\title{
Not all synaesthetes are created equal: Projector versus associator synaesthetes
}

\author{
MIKE J. DIXON, DANIEL SMILEK, and PHILIP M. MERIKLE \\ University of Waterloo, Waterloo, Ontario, Canada
}

\begin{abstract}
In synaesthesia, ordinary stimuli elicit extraordinary experiences. When grapheme-color synaesthetes view black text, each grapheme elicits a photism - a highly specific experience of color. Importantly, some synaesthetes (projectors) report experiencing their photisms in external space, whereas other synaesthetes (associators) report experiencing their photisms "in the mind's eye." We showed that projectors and associators can be differentiated not only by their subjective reports, but also by their performance on Stroop tasks. Digits were presented in colors that were either congruent or incongruent with the synaesthetes' photisms. The synaesthetes named either the video colors of the digits or the colors of the photisms elicited by the digits. The results revealed systematic differences in the patterns of Stroop interference between projectors and associators. Converging evidence from firstperson reports and third-person objective measures of Stroop interference establish the projector/ associator distinction as an important individual difference in grapheme-color synaesthesia.
\end{abstract}

Synaesthesia is a condition in which ordinary stimuli elicit extraordinary experiences. For the synaesthete C.S., pain is orange, and for M.W., the taste of spiced chicken is pointy (Cytowic, 1993). When H.G. hears someone speak, the voice he hears elicits experiences of both color and taste (the first author's voice evoked a taste experience that was "hard to describe, but somewhat like syrup!"). All of these extraordinary experiences reflect variants of synaesthesia. Synaesthesia is rare. Estimates of its incidence range from 1 in 200 people (Ramachandran \& Hubbard, 2001b) to 1 in 2,000 people (Baron-Cohen, Burt, Smith-Laittan, Harrison, \& Bolton, 1996). It runs in families (e.g., both the author Vladimir Nabokov and his mother were synaesthetes) and occurs six times more frequently in females than in males (Baron-Cohen et al., 1996). For synaesthetes such as C.S., M.W., and H.G., the inducing stimulus and the triggered experience cross modalities (e.g., for H.G., sounds trigger colors and tastes). For other synaesthetes, the inducing stimulus and the synaesthetic experience occur in the same modality. For example, in grapheme-color synaesthesia, viewing black digits or letters induces color experiences called photisms.

C. is a 22-year-old undergraduate grapheme-color synaesthete. When she views black digits, her identification of each digit is accompanied by a highly specific color experience (e.g., 2 is red, 7 is yellow). Although different synaesthetes have different grapheme-color pairings, for any given synaesthete, the pairings between

The Natural Sciences and Engineering Research Council of Canada funded this research with operating grants awarded to the first and third authors and a postgraduate scholarship awarded to the second author. Correspondence concerning this article should be addressed to M. J. Dixon, Department of Psychology, University of Waterloo, Waterloo, ON, N2L 3G1 Canada (e-mail: mjdixon@watarts.uwaterloo.ca). graphemes and synaesthetic colors are invariant (BaronCohen, Harrison, Goldstein, \& Wyke, 1993; Svartdal \& Iversen, 1989). Thus, whenever C. views the digit 2, it always elicits a red photism, and when she views the digit 7 , it always elicits a yellow photism and has done so for as long as she can remember.

Grapheme-color synaesthetes also report that whenever they view graphemes, their photisms are elicited independently of their intentions, or in cognitive parlance, automatically (Posner \& Snyder, 1975; Shiffrin \& Schneider, 1977). Previously, we used a variant of the Stroop (1935) task to investigate the automaticity of C.'s photisms (Dixon, Smilek, Cudahy, \& Merikle, 2000). C. was presented with colored digits. The digits were displayed in colors that were either congruent or incongruent with the photisms elicited by the digit. When C. named these colors, she showed a large Stroop effect (i.e., a large difference in response times [RTs] between congruent and incongruent trials). To date, six different studies have shown that synaesthetes demonstrate Stroop effects when they name the colors of congruently or incongruently colored letters or digits (Dixon et al., 2000; Elias, Saucier, Hardie, \& Sarty, 2003; Mattingley, Rich, Yelland, \& Bradshaw, 2001; Mills, Boteler, \& Oliver, 1999; Odgaard, Flowers, \& Bradman, 1999; Wollen \& Ruggiero, 1983). Most investigators agree that these Stroop effects shown by synaesthetes demonstrate that viewing a grapheme automatically elicits an experience of synaesthetic color.

The aim of investigations such as these has been to elucidate those processes that are common to all graphemecolor synaesthetes. The assumption has been that synaesthetic experiences are homogeneous. The subjective reports of synaesthetes we have interviewed, however, have suggested that not all grapheme-color synaesthetes experience synaesthetic colors in a similar fashion. A mi- 
nority of synaesthetes (11 out of 100 in our sample) have described their colors as being "out there on the page," as though a transparency bearing a colored number was placed on top of the written digit. We call these synaesthetes projectors - a label that alludes to their photisms being perceived in external space (see also Cytowic, 1993, 2003). In contrast, the majority of synaesthetes we have interviewed have described their photisms as being "in my mind's eye" or "in my head." Their photisms consist of internally perceived colors, rather than externally perceived colors. Like the photisms of projectors, these internal photisms are both highly specific (e.g., a pale shade of salmon pink) and invariant across a synaesthete's lifetime (e.g., "a 3 has been orange for as long as I can remember"). Their experiences can be likened, at least in part, to that of a nonsynaesthete viewing a blackand-white picture of a stop sign. We "know" the stop sign is a highly specific shade of fire engine red and can readily form an image of this color in our mind's eye, but we do not project this color onto the picture. What differentiates our imagined colors from internal photisms is the automaticity of the processes leading to these experiences. For these synaesthetes, whenever they view a black grapheme, they cannot help but experience an internal photism. We call these synaesthetes associators, because their descriptions of their experiences reflect a strong association between graphemes and specific colors.

In sum, although the language used by different synaesthetes tends to be highly idiosyncratic, their subjective reports suggest an important individual difference: Projectors experience photisms externally, and associators experience photisms internally.

Projected photisms have been shown to attract attention in visual search (Smilek, Dixon, Cudahy, \& Merikle, 2001), enable perceptual grouping (Ramachandran \& Hubbard, 2001a), and induce the McCollough effect (Blake, Palmeri, Marois, \& Chai, in press). For projectors, their photisms have been described as a perceptual reality (Palmeri, Blake, Marois, Flanery, \& Whetsell, 2002). By contrast, the self-reports of associators' lack this projected quality. They experience a highly specific color in their mind's eye, but they do not "see" a red color above the externally presented grapheme.

Such individual differences may have implications for performance on Stroop tasks. When projectors or associators view a colored digit on a video monitor, they experience a Stroop-like stimulus involving two types of colors: the video color on the screen and the photism color induced by the grapheme. In the traditional synaesthetic Stroop task, synaesthetes must ignore their photisms and name the video color as quickly as possible. We hypothesize that projected photisms will be more difficult to ignore than internally experienced photisms. If so, then in a color-naming task, projected photisms would induce larger Stroop effects than would photisms that are experienced internally.

There are several reasons why projected photisms may be more difficult to ignore than internally experienced photisms. Studies using Stroop tasks that vary the spatial proximity between color words and color patches show that irrelevant color words (e.g., RED) are more difficult to ignore when they are presented spatially close to the to-be-named color patches than when they are presented in more spatially disparate positions (Gatti \& Egeth, 1978). Thus, projected photisms may be more difficult to ignore than associated internal photisms because they are perceived as being closer to the video colors that must be named than are internal photisms, which are perceived as being more spatially removed from the video colors. In addition, when a typical projector synaesthete such as $\mathrm{C}$. is presented with a digit in the "wrong" color, she reports that even though she sees both colors, the photism lies above the colored grapheme. Although the colors do not mix and she reports that one color does not occlude the other color, it may be difficult to ignore a percept whose spatial location would normally occlude another object. Associators, by contrast, would not experience a similar problem, because the color they must name and the photism that they must ignore are not perceived as overlapping one another.

A third reason why projected photisms may be more difficult to ignore than associated photisms is that projector synaesthetes may experience their photisms more intensely than do associator synaesthetes. When a subset of synaesthetes were informally asked which led to a more intense experience of color, viewing a color patch or viewing a digit, associator synaesthetes indicated that viewing a color patch yielded a more intense experience of color. The responses of projector synaesthetes were considerably different. For example, C. said that her synaesthetic colors were definitely more intense than the colors induced by color patches. In general, the subjective reports of projectors suggested that projected photisms lead to more intense experiences of color than do internally experienced photisms. In addition, the subjective reports suggested that for projectors, photisms may be more difficult to ignore than video colors, whereas for associators, video colors may be more difficult to ignore than photisms.

To further assess the distinction between projector and associator synaesthetes, we used the traditional synaesthetic Stroop color-naming task, as well as a Stroop photismnaming task. For both tasks, synaesthetes were shown colored graphemes that induced the experience of both video colors and photism colors. In the color-naming task, synaesthetes were told to ignore their photisms and to name the video colors as quickly as possible. In the photism-naming task, synaesthetes were told to ignore the video colors and to name their photisms as quickly as possible. For projectors like C., if photism colors are experienced more intensely than video colors, photism colors should be named relatively quickly and should be relatively immune to interference from the video colors that lie beneath the synaesthetic overlays. Associators, by contrast, should show the opposite pattern of results: If video colors are experienced more intensely than photism colors, it is the video colors that should be named relatively 
quickly and be relatively immune to interference from the internally experienced photisms.

In sum, we expected that projectors and associators would perform the standard synaesthetic Stroop colornaming task differently. Projectors should have more difficulty than associators ignoring their photisms and should, therefore, show larger Stroop effects in color naming. We also expected that projectors and associators would show different patterns of responses when their performance on the color-naming task was contrasted with their performance on the photism-naming task. For projectors, the projected photisms should be hard to ignore in the color-naming task and, thus, should induce large Stroop interference. In contrast, the projected photisms should be named quickly in the photism-naming task and should, therefore, be relatively immune to interference from video colors (i.e., Stroop interference from video colors should be smaller than Stroop interference from photisms). For associators, we expected the opposite pattern of results. Specifically, internally experienced photisms should be easier to ignore than externally presented colors. Thus, unlike for projectors, Stroop interference from video colors should be larger than Stroop interference from photisms for the associator synaesthetes.

In the present experiment, we first classified synaesthetes as projectors or associators on the basis of their subjective reports. Next, we evaluated whether projectors and associators exhibited distinct patterns of performance on the color-naming and photism-naming Stroop tasks. Our goal was to use patterns of Stroop performance to provide objective evidence for the distinction between projectors and associators that heretofore has been based solely on subjective reports.

\section{METHOD}

\section{Participants}

Twelve synaesthetes were classified as either projectors or associators on the basis of their subjective reports. There were 5 projectors ( 4 females, 1 male; mean age $=34$ years) and 7 associators (all females, mean age $=42$ ). All the participants had synaesthesia for letters and digits for as long as they could remember. ${ }^{1}$

\footnotetext{
Apparatus

Eleven participants were tested using a 200-MHz Pentium processor interfaced to a ViewSonic 17PS monitor. The stimuli were presented and RTs were recorded using Micro Experimental Laboratory software (Schneider, 1990). One participant (C.S.) was tested using an Acer computer. For this participant, the stimuli were presented and RTs were recorded using Superlab Pro Experimental Laboratory Software.

\section{Procedure}

Photism-video-color matching. The digits $0-9$ were presented in sequence next to a color pallet composed of 256 colored squares. Synaesthetes selected the square that subjectively best matched the color of their photism for each presented digit. Synaesthetes then rated how closely the selected video color matched the photism for each of the 10 digit-color pairs. On the basis of these ratings, 4 digit-color pairs were selected - those in which the video colors most closely approximated the colors of the synaesthetes' photisms.
}

For 1 participant (C.S.), in order to get the best grapheme-color matches, one digit and three letters were used.

Color-naming task. On each trial, a colored grapheme $(1 \times$ $0.5 \mathrm{~cm}$ ) was presented against a gray background in the center of the video monitor. There were two different types of trials: congruent and incongruent. ${ }^{2}$ On each congruent trial, a grapheme was displayed in the color that corresponded to the color of the synaesthete's photism for that grapheme. There were 120 congruent trials, 30 with each grapheme. On each incongruent trial, a grapheme was displayed in a color that was incongruent with the synaesthete's photisms for that grapheme. There were 360 incongruent trials, 90 of each grapheme. Each grapheme was presented 30 times using each of three incongruent colors. ${ }^{3}$

Following eight practice trials, the congruent and incongruent trials were presented in random order. Graphemes remained on the screen until their colors were named. Synaesthetes were asked to ignore the photisms induced by the graphemes and to name the video colors in which the graphemes were displayed as quickly and accurately as possible. Naming triggered a voice key, which recorded RTs with millisecond accuracy, and the experimenter recorded the accuracy of the synaesthetes' responses.

Photism-naming task. For the photism-naming task, the colored graphemes were identical to those used for the color-naming task. The task for the synaesthetes was to name the color of the photism associated with each grapheme as quickly and as accurately as possible.

The color-naming and photism-naming trials were run in separate sessions, with the color-naming session being administered first.

\section{RESULTS AND DISCUSSION}

\section{Errors}

The percentage of errors in each condition of the experiment is shown in Figure 1. A task (color naming vs. photism naming) $\times$ congruency (congruent vs. incongruent) $\times$ synaesthetic subtype (projectors vs. associators) analysis of variance (ANOVA) revealed only a main effect of congruency $[F(1,10)=13.77, p=.004]$. No other main effects or interactions were significant. Thus, the patterns of errors indicate that the interpretation of the RT data presented below are not compromised by speed-accuracy tradeoffs.

\section{Response Times}

Prior to analyzing the correct RTs, the outliers for each participant were removed using a recursive outlier elimination procedure (Van Selst \& Jolicœur, 1994).

Figure 1 shows the mean RTs for each condition for the 5 projectors (left side of Figure 1) and the 7 associators (right side of Figure 1). RTs were analyzed using a task (color naming vs. photism naming) $\times$ congruency (congruent vs. incongruent) $\times$ synaesthete subtype (projector vs. associator) ANOVA. The analysis revealed a significant task $\times$ congruency $\times$ subtype interaction $[F(1,10)=19.24, p=.001]$.

Color-naming RTs and photism-naming RTs were analyzed separately so that we might understand this interaction and evaluate our predictions. We predicted that when colors were named, the photisms of the projectors would be more difficult to ignore (and would generate larger Stroop effects) than would the internally experienced photisms of the associators. As can be seen by 
COLOR NAMING - (Stroop effect induced by photisms)

PHOTISM NAMING ......(Stroop effect induced by colors)

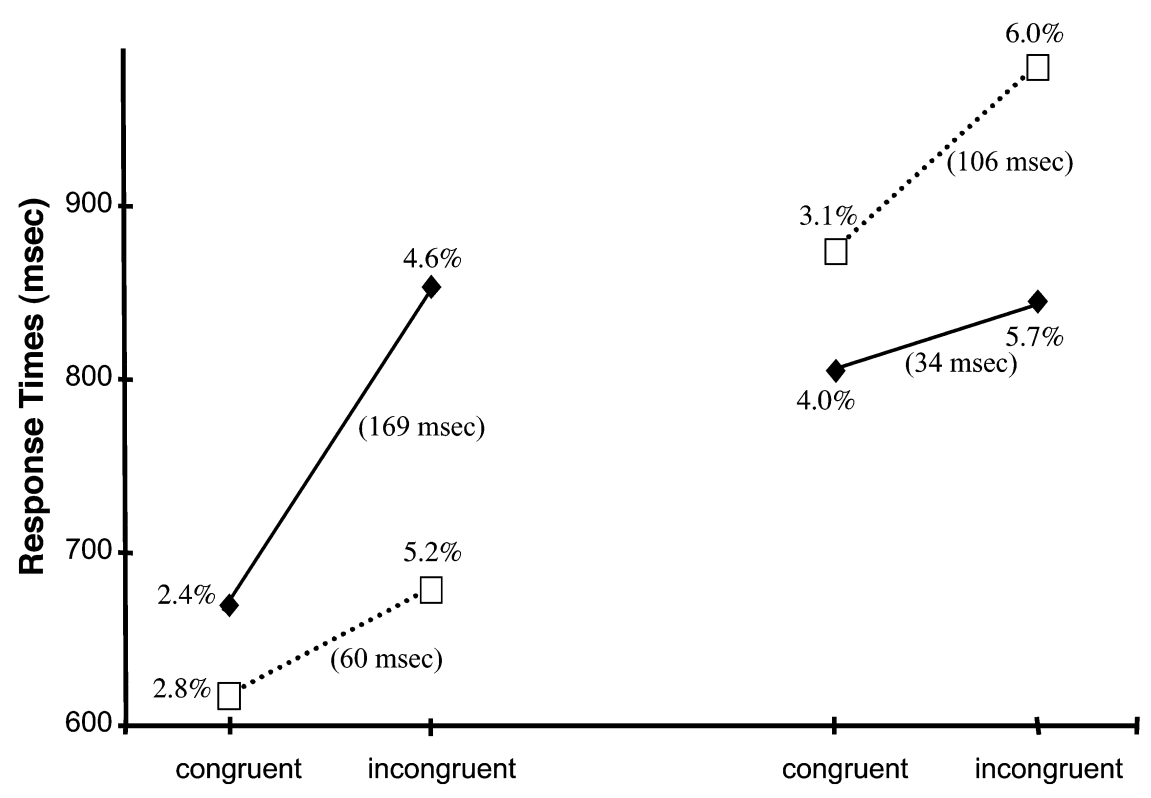

Projector Synaesthetes $(n=5) \quad$ Associator Synaesthetes $(n=7)$

Figure 1. Mean color-naming and photism-naming response times. Percentages of errors appear above the relevant response times. Stroop effects induced by photisms and colors appear in parentheses.

comparing the slopes of the solid lines in Figure 1, for the color-naming task, the projectors showed a significantly larger Stroop effect $(169 \mathrm{msec})$ than did the associators $(34 \mathrm{msec})$, leading to a significant congruency $\times$ synaesthete subtype interaction $[F(1,10)=18.10, p=$ $.002]$. For the photism-naming task, the projectors were faster at naming photisms than were associators $[F(1,10)=$ $9.276, p=.012]$. The congruency $\times$ synaesthete subtype interaction for photism naming, however, was not significant $[F(1,10)=1.016, p=.337]$. That is, there were no significant differences in the sizes of the Stroop effects shown by the projectors and the associators. ${ }^{4}$

The data of the projectors and the associators were also analyzed separately. We predicted that for the projectors, photism naming should be fast and relatively immune to interference from the video colors of the graphemes. As can be seen on the left side of Figure 1, the projectors were faster at naming photisms than they were at naming video colors $[F(1,4)=40.00, p=.004]$, and the difference between the incongruent and the congruent trials on the photism-naming task $(60 \mathrm{msec})$ was much smaller than the difference between the incongruent and the congruent trials on the color-naming task (169 msec), leading to a significant congruency $\times$ task interaction $[F(1,4)=$ $20.52, p=.01]$. These results indicate that the projectors' photisms interfered substantially with their color naming but that their photism naming was relatively im- mune to interference from the colors on the video monitor (i.e., video colors interfered with photism naming much less than photisms interfered with color naming).

The mean RTs for each condition for the associator synaesthetes are shown on the right side of Figure 1. Overall, the associators were faster responding on congruent trials than on incongruent trials $[F(1,6)=10.11$, $p=.019]$. The associators were marginally faster at naming colors than at naming photisms $[F(1,6)=4.31$, $p=.083]$, and they showed more interference from colors than from photisms, leading to a marginal congruency $\times$ task interaction $[F(1,6)=5.74, p=.054]$. These patterns of RTs for the associators were opposite to the patterns of RTs shown by the projectors.

In summary, the results clearly show that projector and associator synaesthetes exhibit different patterns of Stroop interference. The projectors showed larger Stroop effects than did the associators when they attempted to ignore photisms and name video colors. The projectors were also faster at naming photisms than at naming video colors, whereas the associators were marginally faster at naming video colors than at naming photisms. The individual RTs for the projectors and the associators are shown in Table 1. As can be seen in the table, for each projector synaesthete, the Stroop effects induced by the photisms during color naming were at least $40 \mathrm{msec}$ larger than the Stroop effects induced by video colors 
Table 1

\begin{tabular}{|c|c|c|c|c|c|c|c|}
\hline \multicolumn{8}{|c|}{$\begin{array}{l}\text { Mean Individual Response Times for Projectors and Associators on } \\
\text { Congruent (Con) and Incongruent (Inc) Trials and Stroop Effects }\end{array}$} \\
\hline \multirow[b]{2}{*}{ Group } & \multirow[b]{2}{*}{ Age } & \multicolumn{3}{|c|}{ Naming Colors } & \multicolumn{3}{|c|}{ Naming Photisms } \\
\hline & & Con & Inc & $\begin{array}{l}\text { Stroop Effect } \\
\text { (Inc - Con) }\end{array}$ & Con & Inc & $\begin{array}{l}\text { Stroop Effect } \\
\text { (Inc }- \text { Con) }\end{array}$ \\
\hline \multicolumn{8}{|l|}{ Projectors } \\
\hline C. & 22 & 503 & 718 & 215 & 449 & 476 & 27 \\
\hline P4 & 22 & 600 & 712 & 112 & 506 & 531 & 25 \\
\hline P.D. & 50 & 758 & 1,023 & 265 & 672 & 828 & 156 \\
\hline P9 & 45 & 797 & 900 & 103 & 775 & 838 & 63 \\
\hline $\mathrm{P} 102$ & 31 & 736 & 888 & 152 & 657 & 685 & 28 \\
\hline Average & 34 & 679 & 848 & 169 & 612 & 672 & 60 \\
\hline \multicolumn{8}{|l|}{ Associators } \\
\hline E. & 11 & 869 & 921 & 52 & 1,088 & 1,316 & 228 \\
\hline A5 & 43 & 967 & 953 & -14 & 934 & 1,048 & 114 \\
\hline A8 & 58 & 657 & 708 & 51 & 705 & 748 & 43 \\
\hline A19 & $*$ & 645 & 630 & -15 & 966 & 945 & -21 \\
\hline E.H. & 42 & 815 & 910 & 95 & 791 & 890 & 99 \\
\hline T.M. & 39 & 765 & 771 & 6 & 717 & 780 & 63 \\
\hline C.S. & 59 & 869 & 929 & 60 & 933 & 1,151 & 218 \\
\hline Average & 42 & 798 & 832 & 34 & 876 & 983 & 106 \\
\hline
\end{tabular}

*The age of this synaesthete was not made available.

during photism naming (range, 40-188 $\mathrm{msec}$ ). Importantly, not one of the 7 associators that we tested showed this pattern of results. For the associators, either the size of the Stroop effects induced by photisms and colors were similar (A8, A19, E.H.) or colors induced appreciably greater Stroop effects than were induced by photisms (E., A5, T.M., C.S.) - a pattern opposite to that shown by the projector synaesthetes.

The results support the general conclusion that grapheme-color synaesthetes who are classified as projectors or associators on the basis of their subjective reports exhibit distinct patterns of performance on objective measures of grapheme-color synaesthesia. The findings imply that not all grapheme-color synaesthetes experience their synaesthesia in the same fashion and that there are at least two subtypes of grapheme-color synaesthetesprojectors and associators.

\section{GENERAL DISCUSSION}

The present findings provide new insights regarding the automaticity of the processes underlying graphemecolor synaesthesia. Previous studies have demonstrated that synaesthetic colors occur automatically by showing that photisms interfere with color naming (Dixon et al., 2000; Elias et al., 2003; Mattingley et al., 2001; Mills et al., 1999; Odgaard et al., 1999; Wollen \& Ruggiero, 1983). We measured not only the extent to which synaesthetic photisms interfere with color naming, but also the extent to which video color perception interferes with photism naming. MacLeod and Dunbar (1988) used the magnitude of Stroop effects to order different processes on a continuum of automaticity. Because we measured both the Stroop effects induced by photisms and the Stroop effects induced by normal colors, we can use MacLeod and Dunbar's logic to compare the relative au- tomaticity of the processes underlying both synaesthetic and normal color perception, as well as the relative automaticity of projected and internal photisms.

Two findings concerning automaticity emerge. First, for projector synaesthetes, the processes leading to the synaesthetic color experiences are more automatic than the processes leading to normal perception of video colors. This conclusion is based on the finding that projectors exhibit larger Stroop interference effects from photism colors when naming video colors than from video colors when naming photism colors.

The second conclusion regarding automaticity is that the processes leading to photisms were more automatic for projectors than for the associators. This conclusion is based on the finding that Stroop interference from photisms when colors were named was greater for projectors than for associators. We believe that this occurred because photisms that are experienced as external projections of color are more difficult to ignore than photisms experienced as being internal or, in other words, within the "mind's eye."

The present evidence suggests that the processes leading to photisms were more automatic for projectors than for associators. However, it is currently unknown whether these findings reflect differences on a continuum of automaticity or whether there is something categorically different about the synaesthetic experiences of projector and associator synaesthetes that caused these different Stroop results. Conclusions concerning whether the differences between projectors and associators are continuous or categorical will depend on why projector synaesthetes have more difficulty ignoring photisms than do associator synaesthetes.

Projected photisms might be more difficult to ignore than internal photisms because projected photisms lead to a more intense experience of color than do internal 
photisms. Projectors such as C. said that their synaesthetic colors were more intense than the video colors, whereas associator synaesthetes such as C.S. said that the video colors were more intense than their internal photisms. By extension, projected photisms might be more intense than internal photisms. As a cautionary note, some projectors and associators indicated that the two types of color experiences were so different that they were impossible to rate in terms of their relative intensity. Thus, introspection may prove unreliable in estimating the intensity of different color experiences, and more objective measures may be required for research. However, the fact that the projectors showed larger Stroop effects than the associators did when attempting to ignore their photisms provides objective evidence consistent with the idea that projected photisms are more intense and, therefore, more difficult to ignore than internal photisms.

Projected photisms may also be more difficult to ignore than internal photisms because both projected photisms and video colors are experienced in approximately the same spatial location. Since sources of interference are harder to ignore if they are spatially proximate to the stimuli that must be acted on, the projectors might have had more difficulty ignoring their photisms than did the associators.

The spatial separation account cannot explain why the projectors showed greater interference from their photisms than from their perception of video colors. Given that projected photisms and video colors are equally proximate in space, why should photisms generate more interference than video colors? One possible reason is that projected photisms lead to a more intense experience of color than does viewing video colors. A second possibility is that, typically, projected photisms appear above the presented graphemes. Thus, during color naming, it may be very difficult to ignore a percept whose spatial location would normally support occlusion. This may also account for the relative ease with which the projectors were able to name their photisms. In the context of these Stroop tasks, all 5 projectors were faster at naming the colored overlays than they were at naming the video colors underneath these overlays.

In sum, there are a number of possible reasons why projected photisms are more difficult to ignore than internal photisms. Although some of these reasons (e.g., intensity of photisms) may reflect continuous variables leading projectors to be located at the more automatic end of a continuum of automaticity than are associators, other reasons (proximity relations between photisms and colors) may reflect categorical differences between projectors and associators. Furthermore, there may be multiple reasons why projectors find it more difficult to ignore their photisms than to ignore video colors (e.g., photisms occlude colors and/or photisms are more intense than colors). It will require further experimentation to disclose the relative importance of various possible factors in accounting for the different patterns of
Stroop interference shown by projector and associator synaesthetes. What is of ultimate importance in the present study is that these patterns of Stroop interference can be used to objectively differentiate projector and associator synaesthetes.

The finding that there are different subtypes of grapheme-color synaesthetes has important implications for the study of grapheme-color synaesthesia. To date, the results of several studies have shown that synaesthetically induced colors lead synaesthetes to perform tasks involving perceptual grouping (Ramachandran \& Hubbard, 2001a), perceptual crowding (Ramachandran \& Hubbard, 2001b), visual masking (Wagar, Dixon, Smilek, \& Cudahy, 2002), and visual search (Palmeri et al., 2002; Smilek et al., 2001) in ways that are similar to how nonsynaesthetes perform the same tasks when they are shown physically colored stimuli. However, in all studies demonstrating these perceptual effects, the synaesthetes who were tested were synaesthetes whom we would classify as projectors. It is unknown whether associators would show similar perceptual effects for synaesthetically induced colors. Possibly, associators would not show these perceptual effects, because these effects may depend critically on externally projected synaesthetic colors. Unless these critical individual differences among synaesthetes are recognized, inconsistent findings and failures to replicate will likely characterize research concerning grapheme-color synaesthesia.

The differences in the magnitude of the interference from photisms shown by projector and associator synaesthetes suggest that care must be taken when interpreting findings based on group studies of grapheme-color synaesthesia. Mattingley et al. (2001) tested 15 graphemecolor synaesthetes on a synaesthetic Stroop task. These synaesthetes were all associators (Mattingley \& Rich, 2004). Although on average these synaesthetes named the colors of congruently colored stimuli more quickly than they named the colors of incongruently colored stimuli, some of these associators did not demonstrate Stoop effects (Mattingley \& Rich, 2004). Thus, it may well be that only projector synaesthetes will invariably show large photism-induced Stroop effects when naming colors. Caution must therefore be exercised when interpreting Stroop interference as a diagnostic cognitive marker of grapheme-color synaesthesia. ${ }^{5}$

The distinction between projector and associator synaesthetes is important for refining neuropsychological models of grapheme-color synaesthesia (e.g., BaronCohen et al., 1993; Dixon, Smilek, Wagar, \& Merikle, 2004; Ramachandran \& Hubbard, 2001a, 2001b; Smilek \& Dixon, 2002). Such models are informed by studies documenting how nonsynaesthetes process graphemes and colors. Brain imaging and evoked response potentials conducted on patients with chronically implanted electrodes indicate that graphemes are processed by anterior and lateral areas of the fusiform gyrus, as well as by posterior inferior temporal (PIT) areas (Allison, McCarthy, Nobre, Puce, \& Belger, 1994; Cohen \& Dehaene, 
1995; Polk \& Farah, 1998). Posterior to and more medial to these grapheme areas are areas of the fusiform and lingual gyri that are involved in the perception of externally presented colors (e.g., Allison et al., 1994; Chao \& Martin, 1999; Hadjikhani, Liu, Dale, Cavanagh, \& Tootell, 1998; Lueck et al., 1989; McKeefry \& Zeki, 1997; Zeki \& Marini, 1998). Importantly, imaging studies distinguish between brain areas underlying the perception of external color versus areas subserving knowledge about higher order aspects of colors, such as colors perceived on objects and knowledge about the color of objects (Chao \& Martin, 1999; Gulyas, Heywood, Popplewell, Roland, \& Cowey, 1994; Zeki \& Marini, 1998). Of particular relevance to synaesthesia, Chao and Martin had participants name the colors typically associated with common objects (e.g., name colors associated with achromatic pictures of a stop sign, a bulldozer, etc.). This task activated areas that were located slightly lateral to areas that were activated by externally presented colors.

Building on such work, two general classes of models have been proposed to account for synaesthetic photisms: cross-talk models and feedback, or interactive, models. Cross-talk models propose that synaesthetes have abnormal connectivity between different (but often adjacent) brain areas (Ramachandran \& Hubbard, 2001a, 2001b). Feedback models (Dixon et al., 2004; Grossenbacher \& Lovelace, 2001; Smilek \& Dixon, 2002; Smilek et al., 2001) propose that information is processed in a bottom-up fashion but that later stage activation feeds back to activate earlier stages and it is this abnormal feedback that elicits the unusual synaesthetic experiences.

Both cross-activation and feedback may ultimately be necessary to account for different types of graphemecolor synaesthesia. Consider first associator synaesthetes, for whom graphemes elicit internal experiences of colors. A neuropsychological account of such an experience may involve cross-activations between anterior areas of the fusiform that selectively respond to graphemes (Allison et al., 1994) and areas of the fusiform that are associated with knowledge about the color of objects (Chao \& Martin, 1999). Ramachandran and Hubbard (2001b) proposed that "higher" synaesthetes have abnormal cross-talk between the angular gyrus, which processes the concepts of numerals, and the superior temporal gyrus, which processes the conceptual aspects of color. Thus, cross-activation provides a reasonable explanation of the experience of associator (or higher) synaesthetes.

Next, consider projector synaesthetes, for whom graphemes lead to an external experience of color. Ramachandran and Hubbard (2001b) have referred to these synaesthetes as "lower" synaesthetes. They propose that these lower synaesthetes have abnormal hyperconnectivity between areas of the fusiform gyrus that process form and areas of the fusiform (V4) that are associated with color.

Instead of cross-activation, we have proposed that reentrant feedback (Dixon et al., 2004; Smilek \& Dixon, 2002; Smilek et al., 2001) accounts for projected pho- tisms. In our model, information flows along both feedforward and feedbackward connections (e.g., Di Lollo, Enns, \& Rensink, 2000; Grossenbacher \& Lovelace, 2001). When a projector synaesthete such as C. views a black digit, information cascades forward through V1 and V2 to posterior areas of the fusiform gyrus that deal with digit form. Information continues to cascade forward to anterior fusiform and PIT cortical areas, where the presented form is recognized as that of a specific digit or letter (see Allison et al., 1994). Information then cascades back from anterior fusiform and PIT areas to V4/V8, using reentrant pathways. Thus, it is this feedback from later stage areas where forms are recognized as specific graphemes to earlier stage areas that are involved in the perceptual experience of color that accounts for projected photisms.

Evidence from a number of sources is consistent with a reentrant feedback account of projected photisms. Consider a projector synaesthete such as C., who experiences external photisms for both digits and letters. She describes how even if the same letter is presented in markedly different fonts or in cursive script, the synaesthetic overlays will have identical colors. The fact that a myriad of graphemic forms can elicit identical colors is consistent with the idea that it is the identity of the letter (i.e., the meaning of the grapheme) that activates the color, rather than the specific form of the grapheme. In addition, ambiguous graphemes (e.g., a 5 can be interpreted as the digit 5 or the letter S) will automatically elicit different synaesthetic colors, depending on context. That is, exactly the same graphemic form (e.g., 5) will have a brown overlay if it is interpreted as a letter, but a green overlay if it is interpreted as a digit (Myles, Dixon, Smilek, \& Merikle, 2003). Thus, it cannot be the form of the grapheme alone that triggers the colored overlays of projector synaesthetes; later stage areas involving the meaning of the grapheme must ultimately determine the color of the projected photism.

Although the meaning of a grapheme determines the color of a photism, other aspects of projector synaesthetes' experiences implicate the involvement of early visual stages. For example, the tight spatial coupling that occurs between the form of the grapheme and the projected synaesthetic overlay is suggestive of retinotopic early stage visual areas. Also, projected photisms afford perceptual grouping and eliminate perceptual crowding effects (Ramachandran \& Hubbard, 2001a, 2001b), influence visual search (Palmeri et al., 2002; Smilek et al., 2001), and eliminate object substitution masking (Wagar et al., 2002). All of these findings indicate that projected photisms are associated with early stage, perceptual aspects of color, rather than with later stage conceptual aspects of color. To reconcile the late stage influences of meaning and the early stage perceptual reality (Palmeri et al., 2002) of projected photisms, we proposed that graphemic form activates meaning and meaning backactivates early stage visual areas to produce the experience of projected photisms. 
Previously, we suggested that activation from meaning feeds back to such areas as V4/V8 (Dixon et al., 2004; Smilek \& Dixon, 2002). The finding that projected photisms can induce the McCollough effect (Blake et al., in press) suggests that reentrant feedback might activate even earlier color areas, such as V1, in some projector synaesthetes. Although further imaging research will be necessary to establish exactly which visual areas become activated by grapheme-induced photisms, it is imperative that researchers separate projector from associator synaesthetes in the course of conducting such research.

In conclusion, the present study highlights the importance of using both first-person subjective reports of experiences and third-person objective measures to study synaesthesia. The first-person subjective reports were important because they initially suggested the possible distinction between projector and associator synaesthetes. These subjective reports were then used to classify synaesthetes as projectors or associators, to see whether we could support this distinction using thirdperson objective measures. These objective measures involving color naming and photism naming were important in two ways. First, they corroborated the distinction between projector and associator synaesthetes by revealing very different patterns of Stroop interference for each type of synaesthete. Second, these measures allowed us to draw conclusions regarding the relative automaticity of synaesthetic color perception and normal color perception - conclusions that would have been impossible to reach on the basis of subjective reports alone. Ultimately, we believe that it will be such combinations of first- and third-person approaches that will elucidate both the important individual differences among synaesthetes and the general processes underlying synaesthetic experiences.

\section{REFERENCES}

Allison, T., McCarthy, G., Nobre, A., Puce, A., \& Belger, A. (1994). Human extrastriate visual cortex and the perception of faces, words, numbers, and colors. Cerebral Cortex, 5, 544-554.

Baron-Cohen, S., Burt, L., Smith-Laittan, F., Harrison, J., \& Bolton, P. (1996). Synaesthesia: Prevalence and familiarity. Perception, 25, 1073-1079.

BARON-CoHEN, S., HARrison, J., Goldstein, L. H., \& WyKe, M. (1993). Coloured speech perception: Is synaesthesia what happens when modularity breaks down? Perception, 22, 419-426.

Blake, R., Palmeri, T. J., Marois, R., \& CHaI, Y.-K. (in press). On the perceptual reality of synesthetic colors. In L. Robertson \& N. Sagiv (Eds.), Synesthesia: Perspectives from cognitive neuroscience. New York: Oxford University Press.

Chao, L. L., \& Martin, A. (1999). Cortical regions associated with perceiving, naming, and knowing about colors. Journal of Cognitive Neuroscience, 11, 25-35.

COHEN, L., \& DeHAENE, S. (1995). Number processing in pure alexia: The effect of hemispheric asymmetries and task demands. NeuroCase, 1, 121-137.

CYTOWIC, R. E. (1993). The man who tasted shapes. New York: Warner.

Cytowic, R. E. (2003). Synesthesia. A union of the senses. (2nd ed.). Cambridge, MA: MIT Press.

Di Lollo, V., EnNS, J. T., \& Rensink, R. A. (2000). Competition for consciousness among visual events: The psychophysics of reentrant visual pathways. Journal of Experimental Psychology: General, 129, 481-507.
Dixon, M. J., Smilek, D., Cudahy, C., \& Merikle, P. M. (2000). Five plus two equals yellow. Nature, 406, 365 .

Dixon, M. J., SMilek, D., WaGaR, B. M., \& Merikle, P. M. (2004). Grapheme-color synaesthesia: When 7 is yellow and D is blue. In G. A. Calvert, C. Spence, \& B. E. Stein (Eds.), Handbook of multisensory processes (pp. 837-849). Cambridge, MA: MIT Press.

Elias, L. J., Saucier, D. M., Hardie, C., \& Sarty, G. E. (2003). Dissociating semantic and perceptual components of synaesthesia: Behavioural and functional neuroanatomical investigations. Cognitive Brain Research, 16, 232-237.

GATTI, S. V., \& EGETH, H. E. (1978). Failure of spatial selectivity in vision. Bulletin of the Psychonomic Society, 11, 181-184.

Grossenbacher, P. G., \& Lovelace, C. T. (2001). Mechanisms of synesthesia: Cognitive and physiological constraints. Trends in Cognitive Sciences, 5, 36-41.

Gulyas, B., Heywood, C. A., Popplewell, D. A., Roland, P. E., Cowey, A. (1994). Visual form discrimination from color or motion cues: Functional anatomy by positron emission tomography. Proceedings of the National Academy of Sciences, 91, 9965-9969.

Hadjikhani, N., LiU, A. K., Dale, A. M., CavanAGH, P., \& Tootell, R. B. H. (1998). Retinotopy and color sensitivity in human visual cortical area V8. Nature Neuroscience, 1, 235-241.

Lueck, C. J., Zeki, S., Friston, K. J., Deiber, M.-P., Cope, P., Cunningham, V. J., Lammertsma, A. A., Kennard, C., \& Frackowiak, R. S. J. (1989). The colour centre in the cerebral cortex of man. $\mathrm{Na}$ ture, 340, 386-389.

MACLEOD, C. M., \& Dunbar, K. (1988). Training and Stroop-like interference: Evidence for a continuum of automaticity. Journal of Experimental Psychology: Learning, Memory, \& Cognition, 14, 126-135.

MATTINGLEY, J. B., \& Rich, A. N. (2004). Behavioral and brain correlates of multisensory experience in synaesthesia. In G. A. Calvert, C. Spence, \& B. E. Stein (Eds.), Handbook of multisensory processes (pp. 851-865). Cambridge, MA: MIT Press.

Mattingley, J. B., Rich, A. N., Yelland, G., \& Bradshaw, J. L. (2001). Unconscious priming eliminates automatic binding of colour and alphanumeric form in synaesthesia. Nature, 410, 580-582.

McKeefry, D. J., \& ZeKI, S. (1997). The position and topography of the human colour centre as revealed by functional magnetic resonance imaging. Brain, 120, 2229-2242.

Mills, C. B., Boteler, E. H., \& Oliver, G. K. (1999). Digit synaesthesia: A case study using a Stroop-type test. Cognitive Neuropsychology, 16, 181-191.

MY̌les, K. M., Dixon, M. J., Smilek, D., \& Merikle, P. M. (2003). Seeing double: The role of meaning in alphanumeric-colour synaesthesia. Brain \& Cognition, 53, 342-345.

Nunn, J. A., Gregory, L. J., Brammer, M., Williams, S. C., Parslow, D. M., Morgan, M. J., Morris, R. G., Bullmore, E. T., BaronCohen, S., \& Gray, J. A. (2002). Functional magnetic resonance imaging of synesthesia: Activation of V4/V8 by spoken words. $\mathrm{Na}$ ture Neuroscience, 5, 371-375.

OdgaARd, E. C., Flowers, J. H., \& Bradman, H. L. (1999). An investigation of the cognitive and perceptual dynamics of a colour-digit synaesthete. Perception, 28, 651-664.

Palmeri, T. J., Blake, R., MAROIS, R., Flanery, M. A., \& Whetsell, W., JR. (2002). The perceptual reality of synesthetic colors. Proceedings of the National Academy of Sciences, 99, 4127-4131.

Paulesu, E., Harrison, J., Baron-Cohen, S., Watson, J. D. G., Goldstein, L., Heather, J., Frackowiak, R. S. J., \& Frith, C. D. (1995). The physiology of coloured hearing: A PET activation study of colourword synaesthesia. Brain, 118, 661-676.

PolK, T. A., \& FARAH, M. J. (1998). The neural development and organization of letter recognition: Evidence from functional neuroimaging, computational modeling, and behavioral studies. Proceedings of the National Academy of Sciences, 95, 847-852.

PoSNER, M. I., \& SNYDER, C. R. R. (1975). Attention and cognitive control. In R. L. Solso (Ed.), Information processing and cognition: The Loyola symposium (pp. 55-85). Hillsdale, NJ: Erlbaum.

Ramachandran, V. S., \& Hubbard, E. M. (2001a). Psychological investigations into the neural basis of synaesthesia. Proceedings of the Royal Society of London, 268, 979-983.

RAmachandran, V. S., \& Hubbard, E. M. (2001b). Synaesthesia: A 
window into perception, thought and language. Journal of Consciousness Studies, 8, 3-34

SCHNEIDER, W. (1990). MEL user's guide: Computer techniques for real-time experimentation. Pittsburgh, PA: Psychology Software Tools.

Shiffrin, R. M., \& SchneIder, W. (1977). Controlled and automatic human information processing: II. Perceptual learning, automatic attending, and a general theory. Psychological Review, 84, 127-190.

SMILEK, D., \& DixON, M. J. (2002). Towards a synergistic understanding of synaesthesia: Combining current experimental findings with synaesthetes' subjective descriptions. Psyche, 08. Retrieved July 1, 2003 from http://psyche.cs.monash.edu.au/v8/psyche-8-01-smilek.html.

Smilek, D., Dixon, M. J., Cudahy, C., \& Merikle, P. M. (2001). Synaesthetic photisms influence visual perception. Journal of Cognitive Neuroscience, 13, 930-936.

STROOP, J. R. (1935). Studies of interference in serial verbal reactions. Journal of Experimental Psychology, 18, 643-662.

SVARTDAL, F., \& IVERSEN, T. (1989). Consistency in synesthetic experience to vowels and consonants: Five case studies. Scandinavian Journal of Psychology, 30, 220-227.

VAN SELST, M., \& JOLICEUR, P. (1994). A solution to the effects of sample size on outlier elimination. Quarterly Journal of Experimental Psychology, 47A, 631-650.

Wagar, B. M., Dixon, M. J., Smilek, D., \& Cudahy, C. (2002). Colored photisms prevent object-substitution masking in digit-color synesthesia. Brain \& Cognition, 48, 606-611.

Wollen, K. A., \& RUGGIERo, F. T. (1983). Colored-letter synaesthesia. Journal of Mental Imagery, 7, 83-86.

ZEKI, S., \& MARINI, L. (1998). Three cortical stages of colour processing in the human brain. Brain, 121, 1669-1685.

\section{NOTES}

1. Controls were not run, because they have no photisms to name. Training controls to associate graphemes with colors was not feasible, because training would not simulate a synaesthete's lifetime of experiences with grapheme-color pairings. For these reasons, we chose to compare only projector and associator synaesthestes.

2 . The first 2 participants ( 1 projector and 1 associator synaesthete) were administered 108 congruent, 324 incongruent, and 108 baseline trials (colored squares). Problems with finding appropriate baseline trials for the photism-naming task led us to test the remaining participants only on congruent and incongruent trials.

3. Due to a computer error, 1 projector and 2 associator synaesthetes received twice as many trials.

4. In evaluating interaction effects (such as the congruency $\times$ group interactions in this experiment), one would need to be cautious if these interactions coincided with main effects of group, because of potential scaling artifacts. In the photism-naming task, there was a large main effect of group, but no significant interaction. In the color-naming task, there was a significant congruency $\times$ group interaction, but importantly, there was no main effect of synaesthetic group. Thus, the finding that the projectors showed a larger Stroop effect during color naming than did the associators cannot be explained by a scaling artifact.

5. Two other group studies have involved brain imaging of photisms induced by spoken words (Nunn et al., 2002; Paulesu et al., 1995). Photisms induced by auditory stimuli do not map on to the projectorassociator distinction of grapheme-color synaesthetes. The projector C., for example, experiences photisms induced by spoken words in her mind's eye.

(Manuscript received October 15, 2003;

revision accepted for publication June 2, 2004.) 